\title{
Trials for isolation of reticuloendotheliosis virus from commercial chicken flocks for using the isolates in the detection of extraneous virus contamination in live vaccines
}

\author{
Badawi A. A. ${ }^{1 *}$, Salama S. S., and Elham A. Elibiary. \\ ${ }^{1}$ The Central Laboratory for Evaluation of Veterinary Biologics. Abbasia, Cairo, Egypt.
}

\begin{abstract}
In an attempt to isolate reticuloendotheliosis virus (REV) from field cases, plasma of commercial broiler chickens-suspect to have virus infection-were examined. Samples were inoculated in chicken embryo fibroblasts and after proper incubation infected cultures were assayed for REV-antigen by ELISA, immuno-peroxidase (IP) plaque assay, and PCR. Specificity of ELISA and IP was evaluated by comparing their results with that obtained by PCR. REV could be isolated and virus antigen was detected in cell cultures by all three techniques. Results showed that PCR and ELISA are more specific than IP in detection of REV-antigen. However, the sensitivity of ELISA was affected by the criterion used for determination of the cut-off point. Further studies are needed for full characterization of the isolated virus by using reference antiserum or strain specific primers for PCR.
\end{abstract}

Reticuloendotheliosis viruses (REVs) are a group of pathogenic avian type-C retroviruses that induce reticular and lymphoid tumors in different avian hosts including chickens, turkeys, ducks, pheasant and quail. The representative strains of REVs include the defective REV-T; the non defective REV-A, the spleen necrosis virus (SNV), duck infectious anemia (DIA), and chicken syncytial virus (CSV) (Witter and Fadly 2003). Before 1973, the only reported incidence of disease caused by REV was in turkey flock in the USA (Zeigel et al., 1966). Since this time, several reports of REV infection in chicken and turkey flocks in different countries were assigned. This apparent increase in the incidence of REV infections is thought to be a result of widespread use of REV- contaminated vaccines (Nicholas and Thornton, 1983). REV has been isolated from Marek's disease vaccines (Yuasa et al., 1975, Koyama et al., 1976, Jackson et al., 1977, Bagust et al., 1979, and Liu et al., 2009) and from fowl pox vaccines (Bendheim (1973), Fadly et al., (1996), Diallo et al., (1998), and Liu et al., (2009)). The requirements for detection of REV in viral vaccines are described in British Pharmacopoeia (2002) and European Pharmacopoeia (1998) by using in vitro and/or in vivo assays. Until now, routine testing of live

* Corresponding author. Tel.: +2 01066655085; E-mail address: selimsalama2000@yahoo.com (Selim S. Salama). vaccines for detection of REV contamination is faced with some technical obstacles such as the lack of RE virus stock to act as positive control in all biological assays. The objective of this study was an attempt to isolate REV from field samples and assaying the isolated virus by various tests to determine which is the most suitable technique to be used for current testing of vaccines for detection of REV contamination.

\section{Material and Methods}

Cell culture. Secondary chicken embryo fibroblasts (CEFs) were prepared from 9-11 dayold SPF embryos as described by Schat and Purchase (1989). Cells were cultured in MEM supplemented with $5 \%$ calf serum and antibiotics. After $24 \mathrm{hrs}$ of incubation at $37^{\circ} \mathrm{C}$, growth medium was removed and the confluent monolayer was inoculated with test samples then maintenance medium with $2 \%$ serum was added. Chemicals and reagents. Goat anti-rabbit, and goat anti-chicken, HRP conjugated IgG (Sigma) - As a working solution, anti-rabbit conjugate was diluted at 1:1000 in PBS while anti-chicken conjugate was diluted at 1:200 as recommended by the manufactures.

Antisera. Rabbit anti-REV serum and REV monoclonal antibody (mab) 11A25 and 11C237 were kindly provided by DR. A.M. Fadly, USDA, East Lansing, Michigan, USA. Chicken anti-REV serum was obtained from commercial chicken flocks suspect to have REV infection. Chicken serum was tested for REV antibody by 
IDEXX commercial ELISA kit as recommended by the manufacturer. REV antibody-positive sera were further tested for detection of antibody against NDV, IBV, IBDV, AEV or Reoviruses by using Biocheck ELISA Kit. Only serum samples that reacted positive for REV antibody, but not for any other virus were selected. AntiREV chicken serum was pooled, titrated, and used as primary antibody in the immuno peroxidase plaque assay.

\section{Virus Isolation and Identification.}

Plasma was obtained from blood samples collected from chicken flocks suspect to have REV infection. samples were drown from wing vein in $2 \%$ EDTA, centrifuged at $500 \mathrm{xg}$ for 10 min and plasma were separated and stored at $80^{\circ} \mathrm{C}$ until used for RE virus isolation (Davidson et al 1995). Monolayers of secondary CEFs were prepared in 35-mm plastic dishes and in 24-well TC plates. Each plasma sample was inoculated in each of two cultures $(0.5 \mathrm{ml} / \mathrm{dish}$, and 0.1 $\mathrm{ml} /$ well). After adsorption at $37^{\circ} \mathrm{C}$ for $30 \mathrm{~min}$., maintenance medium was added and cultures were maintained at $38^{\circ} \mathrm{C}$. At 6-day PI, cultures grown in dishes were tested by immuno peroxidase plaque assay (IP), while at 7-9 days PI, cultures in plates were subjected to 2 cycles of freezing and thawing and cell lysates were stored at $-20{ }^{\circ} \mathrm{C}$ until used for assaying REV antigen by ELISA. Live vaccines of Newcastle disease (ND), infectious bronchitis (IB), infectious bursal disease (IBD), chicken anemia (CA), and Marek's disease (MD) viruses were reconstituted in sterile distilled water, passed through $0.22 \mu$ membrane and diluted in TC medium. Each of the above vaccines was assayed for REV as described for plasma and they acted as controls to check specificity of the experiments. In all attempts, uninoculated cultures were maintained as negative controls.

Specificity of ELISA and IP for detection of REV.

Samples that have been found REV-positive or suspect positive by ELISA and IP were reexamined by PCR. DNA from some selected samples were extracted and tested by PCR for detection of REV sequences using a pair of primers designed for the spleen necrosis virus (SNV) LTR.

Sensitivity of ELISA, IP and PCR for detection of REV antigen.

The REV-positive samples were serially diluted from $10^{-1}$ to $10^{-7}$ and each dilution was inoculated in each of 4 replicates of CEF cultures. After incubation at $37^{\circ} \mathrm{C}$ for proper time, the infected cultures were tested by ELISA, IP and PCR to determine the least dilution of the virus that could be detected by each test.

Virus detection by ELISA. The procedures described by Cui et al., (1988) and Witter (1989) for detection of REV antigen by ELISA was followed. Briefly, 100 $\mu 1 /$ well of cell lysates was added to microtiter ELISA plates (Nunc) that were coated with a mixture of monoclonal antibody (mab) 11A25 and 11C237 each diluted 1:1000 in carbonate buffer. The REV antigen was detected using rabbit anti-REV serum and HRP conjugated goat anti-rabbit IgG diluted 1:1000 in BPS.

Immunoperoxidase plaque assay for REV: The test was performed as described by Calvert and Nazerian (1994). Briefly, CEF cultures grown in $35-\mathrm{mm}$ dishes were inoculated with various test samples, then TC medium with $0.6 \%$ agar was added and cultures were incubated at $37^{\circ} \mathrm{C}$ for 6 days when medium and agar overlay was removed and cell layer was fixed with acetone/ethanol 3:2 and chicken antiREV serum diluted 1:100 in PBS with 3\% bovine serum albumin was added for 1 hour at room temp. After washing, HRP-conjugated goat anti chicken IgG diluted 1:200 in BPS was added for 1 hour. Substrate solution composed of $\quad 0.6 \mathrm{mg} / \mathrm{ml} \quad$ diaminobenzidine tetrahydrochloride (sigma), $45 \mathrm{mM}$ Tris $\mathrm{HCl}$, $0.03 \%$ cobalt chloride (sigma) and $0.03 \% \mathrm{H}_{2} \mathrm{O}_{2}$ was added for $10 \mathrm{~min}$ and plaques were counted in inverted position.

Detection of REV by PCR: DNA was extracted from CEFs infected with various test samples and PCR was used to detect REV sequences as described by Aly et al., (1993). The primers used were based on sequences of the proviral SNVLTR. The direct primer was CAT ACT GGA GCC AAT GGT G-3' and the reverse primer was 5' AAT GTT GTA GCG AAG TAC T-3'. The expected amplified DNA band size is 291 bp. Cycling parameters used were $94{ }^{\circ} \mathrm{C}$ for 2 min. for initial denaturation, followed by 30 cycles at $94{ }^{\circ} \mathrm{C}$ for $1 \mathrm{~min}$., $55^{\circ} \mathrm{C}$ for $2 \mathrm{~min}$., and $72{ }^{\circ} \mathrm{C}$ for $1 \mathrm{~min}$. A final elongation proceeded at $72{ }^{\circ} \mathrm{C}$ for $6 \mathrm{~min}$. Mixtures lacking template were routinely included as negative control, while samples extracted from cultures infected with NDV,IBV,IBDV,CAV, and MDV were used to check the specificity of the test.

\section{Results}

(1) ELISA for REV antigen: The average background absorbance obtained when more 
than 30 uninfected control cultures were tested was 0.274 and one standard deviation above the control was 0.1(Data not shown). The cut-off value was calculated as the mean absorbance of negative control plus 2.5 times the standard deviation. Consequently, samples that produced an absorbance greater than 0.524 was scored positive. Results in table 1 shows that one plasma sample out of 38 was positive for REV antigen. Although REV antigen was detected in one plasma sample (No. PL-05), it was also detected in one uninfected culture (No. Un-07) and in one MDV-infected culture (No. MD-02). The absorbance of uninfected and MDVinfected cultures was 0.524 , and 0.528 , respectively compared to 0,669 in plasma infected culture. This mean that both the uninfected and MDV-infected cultures lies on the border of positive while the plasma sample is highly positive (the cutoff point $=0.524$ ). Data also shows that the least absorbance was recorded in IBV-infected culture (0.268), while the highest absorbance was obtained in plasma infected cultures.

(2) Plaque assay for REV: The immunoperoxidase technique was used for detection of plaque in CEFs infected with various test samples. Preliminary trials for detection of REV by immunoperoxidase (IP) revealed considerable high background and dark plaques in some dishes when chicken serum was used at dilution 1:10(data not shown). Therefore, to reduce the likelihood of obtaining non specific reactions serum was used in all subsequent trials at 1:100. Results of IP plaque assay was shown in table 1. Plaques were detected in 3 out of 38 plasmainfected cultures (No. PL-05, PL-13, and PL27). Similar foci were also detected in two out of 11 uninfected cultures (No. Un.-03, and Un-11) and in one CAV-infected culture (No. CA-01). No foci were detected in control cultures infected with any of MDV, NDV, IBV, or IBDV.

\section{Table1: Results of ELISA, Immunoperoxidase (IP) plaque assay and PCR for detection of REV in CEF cultures infected with various test samples.}

\begin{tabular}{|c|c|c|c|c|c|}
\hline \multicolumn{2}{|c|}{ Cultures } & \multicolumn{4}{|c|}{ Results } \\
\hline \multirow{2}{*}{ Treatment } & \multirow{2}{*}{ No. tested } & \multicolumn{2}{|c|}{ ELISA* } & \multirow[t]{2}{*}{ IP } & \multirow[t]{2}{*}{ PCR } \\
\hline & & OD** & Status(+/-) & & \\
\hline \multirow{4}{*}{ 1. Plasma infected } & 35 & $0.335-$ & - & - & $\mathrm{NT}^{* * *}$ \\
\hline & 1(PL-05) & 0.501 & + & + & + \\
\hline & $1(\mathrm{PL}-13)$ & 0.669 & - & + & - \\
\hline & 1(PL-27) & $\begin{array}{l}0.511 \\
0.472\end{array}$ & - & + & - \\
\hline \multirow{4}{*}{ 2.Uninfected } & 8 & $0.311-0.441$ & - & - & - \\
\hline & $1(\mathrm{Un}-03)$ & 0.433 & - & + & - \\
\hline & $1(\mathrm{Un}-07)$ & 0.524 & + & - & - \\
\hline & 1(Un-11) & 0.398 & - & + & - \\
\hline 3. Controls & & & & & \\
\hline a. NDV-infected & 2 & $0.291,0.332$ & - & - & - \\
\hline b. IBV-infected & 2 & $0.268,0.297$ & - & - & - \\
\hline \multirow[t]{2}{*}{ c. MDV-infected } & 1(MD-01) & 0.363 & - & - & - \\
\hline & 1(MD-02) & 0.528 & + & - & - \\
\hline d. IBVD-infected & 2 & $0.315,0.423$ & - & - & - \\
\hline \multirow[t]{2}{*}{ e. CAV-infected } & $1(\mathrm{CA}-01)$ & 0.278 & - & + & - \\
\hline & $1(\mathrm{CA}-02)$ & 0.386 & - & - & - \\
\hline Total & 59 & & $3 / 59$ & $6 / 59$ & $1 / 24$ \\
\hline
\end{tabular}

$*$ mean neg. cont. absorbance $=0.274$, the SD of neg. cont. $=0.1$, the cutoff point $=0.524$.

$* * \mathrm{OD}=$ optical density.

Indistinct results were recorded in some samples by ELISA and IP in exp.1. To verify these results and to check the specificity of both tests in detection of REV, PCR was performed on some selected samples. The selected samples included all uninfected cultures (11 samples), control cultures (10 samples) as well as 3 plasma

$$
* * * \mathrm{NT}=\text { Not tested. }
$$

samples that gave an indistinct result in Exp. 1. As shown in Table (1). One sample ( PL-05) out of 24 was PCR positive for REV. The same sample was positive by ELISA and IP in Exp (1). No PCR product was detected in any of |uninfected or control cultures (Photo 1). 


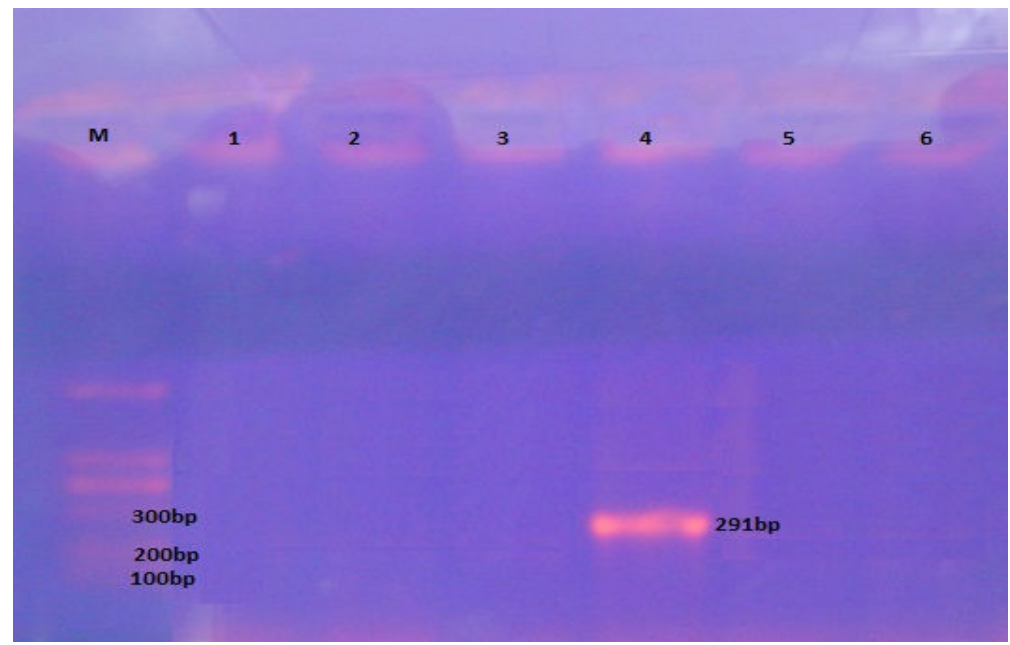

Photo (1): Results of PCR for detection of REV virus in selected cultures. (M :100 bp DNA marker, Lane (1): Uninfected culture, Lane (2): Sample PL-07, Lane (3): Sample PL-13, Lane (4): Sample PL-05, Lane (5): NDV- infected culture and Lane (6): MD- infected culture.)

Plasma sample no. PL-05 that gave positive REV results by each of ELISA, IP, and PCR was serially diluted in TC medium, each dilution was inoculated in each of 4 replicates of CEFs and cultures were assayed for determination of the endpoint of REV antigen that could be detected by each of the three techniques. Results in table
2 shows that the highest limit of detection was recorded by PCR at $10^{-5}$, followed by ELISA at $10^{-3}$, while the detection limit of IP was $10^{-2}$. The titer of the isolated virus was $10^{3.5}, 10^{2.5}$, and $10^{2.3}$ TCID50 by PCR, ELISA \& IP respectively.

Table 2: Limits of sensitivity for detection of REV in CEFs by using ELISA, IP, and PCR.

\begin{tabular}{ccccccc}
\hline \multirow{2}{*}{ Virus dilution } & \multicolumn{5}{c}{ Test used } \\
\cline { 2 - 6 } & \multicolumn{2}{c}{ ELISA } & \multicolumn{2}{c}{ IP } & \multicolumn{2}{c}{ PCR } \\
\cline { 2 - 6 } & +ve/Tested & Titer & +ve/ Tested & Titer & +ve/ Tested & Titer \\
\hline $\mathbf{1 0}^{-1}$ & $4 / 4$ & & $4 / 4$ & & $4 / 4$ & \\
$\mathbf{1 0}^{-2}$ & $2 / 4$ & & $3 / 4$ & & $3 / 4$ & \\
$\mathbf{1 0}^{-3}$ & $2 / 4$ & & $0 / 3$ & & $3 / 4$ & \\
$\mathbf{1 0}^{-4}$ & $0 / 3$ & $10^{2.5}$ & $0 / 4$ & $10^{2.3}$ & $1 / 3$ & $10^{3.5}$ \\
$\mathbf{1 0}^{-5}$ & $0 / 4$ & & $0 / 4$ & & $1 / 4$ & \\
$\mathbf{1 0}^{-6}$ & $0 / 3$ & & $0 / 3$ & & $0 / 4$ & \\
$\mathbf{1 0}^{-7}$ & $0 / 4$ & & $0 / 4$ & & $0 / 3$ & \\
\hline
\end{tabular}

Detection of antibody by ELISA. Chicken sera obtained from suspected flocks were tested at 1:500 dilutions for detection of REV antibody. Interpretation of results was performed by calculation of sample to positive $(\mathrm{S} / \mathrm{P})$ ratio and samples with $\mathrm{S} / \mathrm{P}$ ratio more than 0.5 is considered positive as recommended by the manufacturer. Data in table 3 shows that 23 sample out of $108(21.3 \%)$ were positive for REV antibody. The S/P ratio of positive samples ranged from 0.55 to 1.5 . All REV antibody- positive sera (23 samples) were subsequently tested by ELISA to exclude other antibodies against other viruses that may co-exist with REV. As shown in Table (3) antibody against NDV, IBV, and IBDV were detected in 10, 14, and 14 samples, respectively. These samples were excluded. In some cases antibody against more than one virus was detected in the same bird. No antibody against Reovirus or AE virus was detected. 
Table (3): Results of ELISA for detection of antibody against REV and other viruses in chicken serum.

\begin{tabular}{|c|c|c|c|c|c|c|c|c|c|}
\hline \multicolumn{2}{|c|}{ Chickens } & \multicolumn{3}{|c|}{ REV antibody } & \multicolumn{5}{|c|}{ Antibody against } \\
\hline \multirow[t]{2}{*}{ Flock no. } & \multirow[t]{2}{*}{ No. tested } & \multirow[t]{2}{*}{$\mathrm{S} / \mathrm{P}$ ratio } & \multicolumn{2}{|c|}{ Status } & \multirow{2}{*}{$\begin{array}{c}\text { ND } \\
\text { V }\end{array}$} & \multirow[t]{2}{*}{ Reo } & \multirow[t]{2}{*}{ IBV } & \multirow{2}{*}{$\begin{array}{c}\text { IBD } \\
\text { V }\end{array}$} & \multirow[t]{2}{*}{ AEV } \\
\hline & & & + & - & & & & & \\
\hline \multirow[t]{3}{*}{ I. } & 29 & $0.16-0.41$ & 0 & 29 & $\mathrm{NT}^{*}$ & NT & NT & NT & $\overline{\mathrm{NT}}$ \\
\hline & 6 & $0.55-0.88$ & 6 & 0 & $3 / 6^{*}$ & $0 / 6$ & $6 / 6$ & $4 / 6$ & $0 / 6$ \\
\hline & 1 & 0.92 & 1 & 0 & $\begin{array}{c}* \\
0 / 1\end{array}$ & $0 / 1$ & $0 / 1$ & $0 / 1$ & $0 / 1$ \\
\hline \multirow[t]{3}{*}{ II. } & 32 & $0.19-0.38$ & 0 & 32 & NT & NT & NT & NT & NT \\
\hline & 5 & $0.73-0.92$ & 5 & 0 & $2 / 5$ & $0 / 5$ & $5 / 5$ & $4 / 5$ & $0 / 5$ \\
\hline & 1 & 1.5 & 1 & 0 & $0 / 1$ & $0 / 1$ & $0 / 1$ & $0 / 1$ & $0 / 1$ \\
\hline \multirow[t]{3}{*}{ III. } & 24 & $0.21-0.39$ & 0 & 24 & NT & NT & NT & NT & NT \\
\hline & 7 & $0.68-0.98$ & 7 & 0 & $5 / 7$ & $0 / 7$ & $3 / 7$ & $6 / 7$ & $0 / 7$ \\
\hline & 3 & $0.61-1.16$ & 3 & 0 & $0 / 3$ & $0 / 3$ & $0 / 3$ & $0 / 3$ & $0 / 3$ \\
\hline Total & 108 & & 23 & 85 & 10 & 0 & 14 & 14 & 0 \\
\hline
\end{tabular}

\section{Discussion}

Plasma of commercial broiler chickens suspected to have REV infection was used for virus isolation. Samples were inoculated in CEFs cultures derived from SPF embryos and REV antigen was assayed by ELISA, IP, and PCR. A series of uninoculated cultures as well as cultures inoculated with various live viral vaccines were maintained in parallel to check specificity of the experiments.

Assaying for REV antigen by ELISA and IP revealed an accordance of $88.1 \%$ inbetween both tests. A discrepancy was recorded in 7 samples ( 2 plasma-infected plus 5 control cultures), Table (1). This discrepancy may be attributed to nonspecific reactions. Similar nonspecific reactions were recorded by Nicholas and Thornton (1983) and by Calvert and Nazerian (1994) in detection of REV by Immunoperoxidase plaque assay. They attributed the nonspecific reaction to a variety of factors including the low titer of REV used in the experiments, the fixative used, and the commercial source of primary and secondary antibody.

Using of chicken anti-REV serum as primary antibody in Immunoperoxidase assay may represent another cause of nonspecific reaction. Since serum samples were obtained from commercial flocks, so the likelihood of obtaining nonspecific reaction is not excluded. Although sera were screened for antibody against some viruses as NDV, IBV, IBDV, $\mathrm{AEV}$, and reovirus but this doesn't mean absence of antibody against other pathogens that may interfere with test results. The use of monoclonal antibody (mab) or REV-monospecific antiserum or REV reference serum may overcome these misleadings.

To asses the specificity of ELISA and IP for detection of REV antigen, all samples with doubt or indistinct results were selected and reexamined by PCR. The selected samples included 3 plasma-infected cultures and 21 control cultures. Results of PCR showed that only one out of 24 selected samples was positive. This PCR-positive sample (No. PL-05) was also positive by ELISA and IP in exp.1. No PCR product was detected in all control cultures. These results indicate that PCR is more specific than ELISA and IP in detection of REV. Also indicates that the indistinct results obtained by ELISA and IP were attributed to nonspecific reactions.

Comparing results of ELISA with that of PCR revealed an accordance of $83.3 \%$ inbetween both tests. The difference between ELISA and PCR in detection of REV in CEF cultures may be attributed to the criterion used for interpretation of ELISA results. In our study we used a cut-off point equal to the mean absorbance of negative control plus 2.5 times the standard deviation as recommended by Cui et al., (1988) and by using this criterion, 3 samples were scored positive by ELISA versus 1 sample positive by PCR. The ELISA positive samples were no. PL-05, MD-02, and un-07, while sample no. PL-05 was positive by PCR. When the criterion of cutoff was corrected to be equal to twice the mean absorbance of negative control 
as recommended by Voller (1980), samples no. MD-2 and un-7 were scored negative and sample No. PL-05 is still positive. These results indicated that by using the criterion described by Voller (1980) for determination of ELISA cutoff point, the results of ELISA is comparable with that of PCR and an accordance of $100 \%$ was obtained in-between both techniques.

A number of false-positive results were recorded by Smith and Witter (1983) when they used a cutoff point equal to the mean of negative control plus 2 standard deviation in detection of REV antigen by ELISA. They added that the criterion for interpretation of ELISA results depend on the absorbance cutoff and this point frequently represents a compromise between specificity and sensitivity. Low absorbance threshold increase the probability of increasing false-positive samples. Conversely, sensitivity is compromised if the threshold is set too high.

The PCR results indicated that the test has been shown to be a sensitive and specific test for detection of REV in infected CEFs cultures. REV could be detected with PCR at $10^{-5}$ dilution, compared to $10^{-3}$ for ELISA and $10^{-2}$ for IP.

In this study we used the PCR to detect REV in field samples by using the primers published by Aly et al., (1993), who analyzed five prototypes of REVs. By using the same primers Davidson et al., (1995) could identify 8 REVs isolates and they stated that nucleotide sequence of the primer of REV-LTR chosen by Aly et al., (1993) are universally applicable. Therefore, further studies is needed for characterization of our REV isolate by using either strain specific reference antiserum or a pair of primers designed to identify specific virus strains.

\section{References}

Aly, M. M.; Smith, E. J.; and Fadly, A. M. (1993): Detection of Reticuloendotheliosis virus infection using the polymerase chain reaction. Avian Pathol., 22:543-554.

Bagust, T. J.; Grimes, T. M.; and Dennett, D. P. (1979): Infection studies on a reticuloendotheliosis contaminant of a commercial marek's disease vaccine. Aust. Vet. J., 55:153-157.

Bandheim, U. (1973): A neoplastic disease in turkeys following fowl pox vaccination. Refuah veterinaria 30:3541.

British Pharmacopoeia (Veterinary) (2002): Appendix XVIB (Vet) A19.

Calvert, J. G. and Nazerian, K. (1994): An Immunoperoxidase plaque assay for reticuloendotheliosis virus and its application to a sensitive serum neutralization assay. Avian Dis. 38:138-171

Cui, Z.; Lee, L. F.; Smith, E. J.; Witter, R. L. and Chang, T. S. (1988): Monoclonal-antibody-mediated enzyme-linked immunosorbent assay for detection of reticuloendotheliosis viruses. Av.Dis. 32: 32-40.

Davidson, I.; Borovskaya, A.; Perl, S.; and Malkinson, M. (1995): Use of the polymerase chain reaction for the diagnosis of natural infection of chickens and turkeys with marek's disease virus and reticuloendotheliosis virus. Av. Pathol. 24:69-94.

Diallo, I. S.; Mackenzie, M. A.; Spradbrow, P. B. and Robinson, W. F. (1998): Field isolates of fowl pox virus contaminated with reticuloendotheliosis virus. Avian Pathol., 27:60-66.

European Pharmacopoeia 1998. $3^{\text {rd }}$ ed.

Fadly, A. M. .; Witter, R. L.; Smith, E. J.; Silva, R. F.; Reed, W. M.; Hoerr, F. J. and Putnam, M. R. (1996): An outbreak of lymphomas in commercial broiler breeder chicken vaccinated with fowl pox vaccine contaminated with Reticuloendotheliosis virus. Avian Pathol., 25:35-47.

Jackson, C. A.; Dunn, S. E.; Smith, D. I.; Gilchrist, P. T. and Mac Queen, P. A. (1977): Proventriculitis, 'Nakanue' and reticuloendotheliosis in chickens following vaccination with herpes virus of turkeys (HVT). Aust. Vet. J., 53:457459.

Koyama, H.; Suzuki, Y.; Ohwada, Y. and Saito, Y. (1976): Reticuloendotheliosis group virus pathogenic to chicken isolated from material infected with turkey herpes virus (HVT). Avian Dis., 20: 429-434.

Liu, Q.; Zhao, J.; Su, J.; Pu, J.; Zhang, G. and Liu, J., (2009): Full genome sequences of two reticuloendotheliosis viruses contaminating commercial vaccines. Avian Dis., 53: 341-346.

Nicholas, R. A. J. and Thornton, D. H. (1983): Relative efficiency of techniques for detecting avian Reticuloendotheliosis virus as a vaccine contaminant. Res. Vet. Sci., 34: 377-379.

Schat, K. A., and Purchase, H. G. (1989): Cell culture methods in: a laboratory manual for the isolation and identification of avian pathogens. $3^{\text {rd }}$ ed. pp.167-175.

Smith, E. J. and Witter, R. L. (1983): Detection of antibodies against reticuloendotheliosis viruses by an enzyme-linked immunosorbent assay. Avian Dis., 27: 225234.

Voller, A. (1980): Heterogeneous enzyme-immuno-assays and their applications. In enzyme immunoassays.E.T.Maggio, ed. CRC Press Inc, Boca Raton, Florida pp. 181-195.

Witter, R. L. (1989): Reticuloendotheliosis. In: a laboratory manual for the isolation and identification of avian pathogens. $3^{\text {rd }}$ ed. pp. 143-148.

Witter, R. L., and Fadly, A. M. (2003): Reticuloendotheliosis. In diseases of poultry, $11^{\text {th }}$ ed. Saif, Y.M., J. Barnes, J. Glisson, L. Macdougal, and D. Swyne, eds. Iowa State Univ. Press, Ames, pp. 517-535.

Witter, R. L., and Salter, D. W. (1989): Vertical transmission of reticuloendotheliosis virus in breeder turkeys. Av. Dis. 33: 226-235.

Yuasa, N.; Yoshida, I.; Taniguchi, T.; Kawamura, H.; Wakabayashi, T.; Yamaguchi, S.; Takayanagi, N.; Sato, S.; Sekiya, S. and Horiuchi, T. (1975): Contamination of marek's disease vaccine with REV group virus. Proceedings of the $79^{\text {th }}$ Annual Meeting of the Japanese Society of Veterinary Science. English Summaries pp. 8-10 Zeigel, R. F.; Theileu, G. H.; Twiehaus, M. J. (1966): Electron microscopic observations on RE virus (strain T) that induces reticuloendotheliosis in turkeys, chickens, and Japanese quail. J. Nat. Cancer Ins., 37:709-729. 


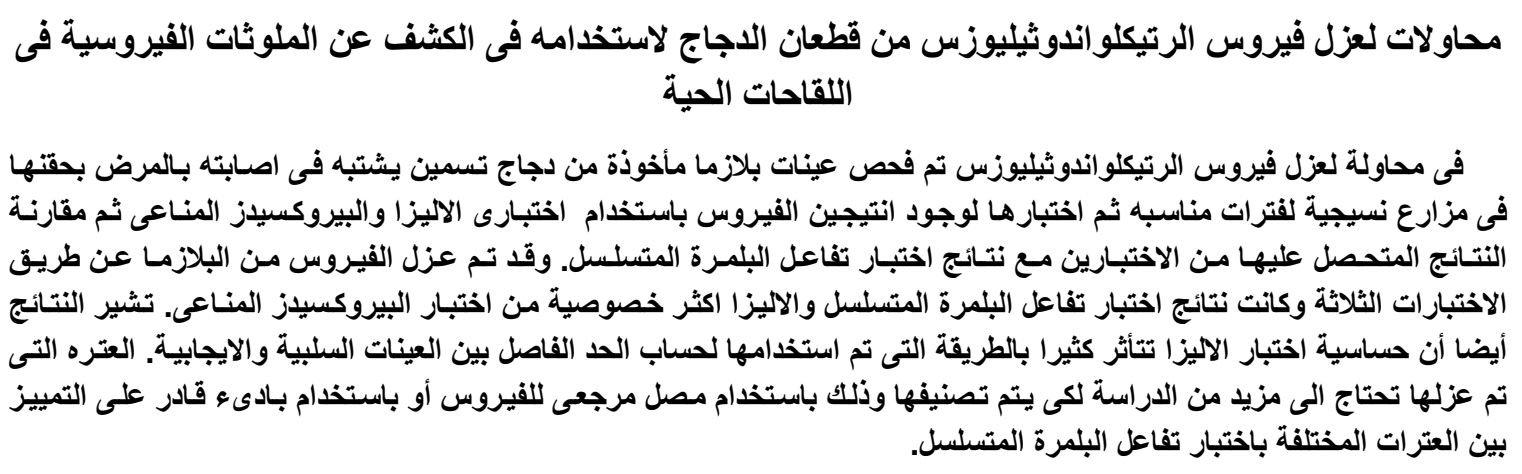

